\title{
A fatal unsuspected case of acquired A hemophilia. Misleading role of therapy with acetylsalicylic acid?
}

\author{
Luca Puccetti $^{1}$ (1) $\cdot$ Francesca Bacchiarri $^{1} \cdot$ Paola Calzoni ${ }^{2} \cdot$ Adele Santoni $^{1} \cdot$ Monica Bocchia $^{1}$
}

Received: 11 March 2021 / Accepted: 10 May 2021 / Published online: 7 June 2021

(c) Società Italiana di Medicina Interna (SIMI) 2021, corrected publication 2021

\section{Dear Editor,}

Acquired hemophilia is a rare disease resulting from autoantibodies (inhibitors) against coagulation factors that leads to bleeding. The most common form is against factor VIII, named acquired A hemophilia (AHA). It occurs mostly in elderly but can be associated with pregnancy and autoimmune disease in youngsters, about $50 \%$ of cases are considered idiopathic, but AHA is also correlated with malignancies and infections [1]. Bleeding diathesis can vary from minor tendency to major and sometimes lethal forms.

Here we report the case of a 73-years old woman, who was admitted to the Emergency department of a spoke hospital during the COVID-19 pandemic emergency, for a large and painful hematoma of the right arm.

The hematoma developed spontaneously in the previous $24 \mathrm{~h}$ and without concomitant or preceding events before the clinical evaluation and reached rapidly the extension reported in Fig. 1. The general practitioner once evaluated by telemedicine the extension of the hematoma, suggested to reach the Emergency Department for specific evaluation.

The patient had no previous history of bleeding tendency, and was treated for 3 years with acetylsalicylic acid (ASA) $100 \mathrm{mg} /$ day, atorvastatin $20 \mathrm{mg} /$ day, valsartan $80 \mathrm{mg} /$ day after a transient ischemic attack (TIA).

After clinical evaluation, no blood exams were done and the patient was discharged from the Emergency Department with the diagnosis of "hematoma of the right arm in patient treated with ASA".

Luca Puccetti

luca.puccetti@unisi.it

1 Hemathology Unit/Coagulative Disorders, "Santa Maria Alle Scotte" University Teaching Hospital of Siena, University of Siena, Siena, Italy

2 Coagulative Disorders Laboratory Unit, Santa Maria Alle Scotte University Teaching Hospital of Siena, Siena, Italy
Therefore, the patient was advised to stop ASA for 3 days and to reach again the hospital in case of further spontaneous hematomas.

Six days later she developed sudden unconsciousness at home and was taken to the nearest Hospital 30 min later. She was admitted at the Emergency department with a 3 point Glasgow Coma Scale (GCS), blood pressure 90/60 mmHg, regular heart rate $104 / \mathrm{min}, \mathrm{SaO} 292 \%$ without oxygen supplementation, respiratory rate $19 / \mathrm{min}$. Clinical examination showed the previously described hematoma (Fig. 1) and various smaller hematomas in the other arm, trunk and limbs.

An electrocardiography (EKG) showed a sinus tachycardia and diffuse negative $\mathrm{T}$ waves in III inferior, aVL lateral and $\mathrm{V}_{4}-\mathrm{V}_{6}$ leads. Blood samples including arterial blood analysis, were immediately taken.

Blood examinations showed a normal blood count with white blood cells (WBC) 9600/ $\mu \mathrm{L}$ (Neutrophils 55\%, Lymphocytes $34 \%$, Monocytes 6\%, Eosinophils 3\%, Basophils $2 \%$,), severe microcytic anemia (hemoglobin $6.4 \mathrm{~g} / \mathrm{dL}$, Hct $29 \%$, MCV $73.6 \mathrm{fL})$ normal platelets value $\left(216,000 \times 10^{3} /\right.$ $\mu \mathrm{L})$, creatinine $1.1 \mathrm{mg} / \mathrm{dL}$, negative troponin I, prothrombin time (PT) $88 \%$ (normal range 75-100\%), INR 1.11 (normal range $<1.2$ ), activated partial thromboplastin time (aPTT) $96 \mathrm{~s}$ (normal range 20-35 secs), aPTT ratio 2.9 (normal range $<1.5$ ), fibrinogen $276 \mathrm{mg} / \mathrm{dL}$ (normal range $150-400 \mathrm{mg} / \mathrm{dL})$.

While the patient was conducted to perform a Computed Tomography (CT) cerebral scan 15 min after the admission in the emergency room, she experienced a cardiac arrest and resuscitation maneuvers were ineffective. Autopsy was requested.

In the meantime, due to the aPTT levels a mixing text was performed alongside with a research for lupus anticoagulant (LAC). LAC was negative while the presence of a circulating inhibitor with levels of $0.8 \%$ activity for Factor VIII were established (normal range 60-120\%). The amount of available blood did not allow quantification of circulating inhibitor titer in Bethesda units. 


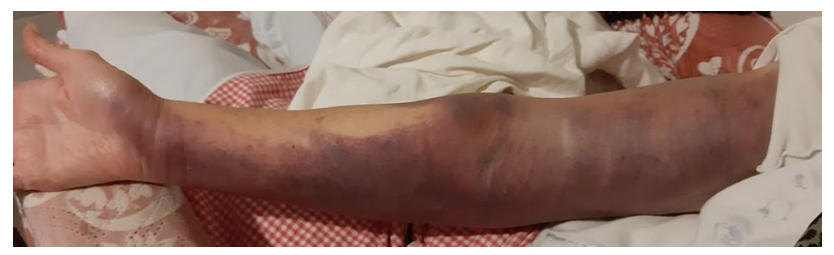

Fig. 1 .

The post-mortem diagnosis of acquired AHA was strongly suggested. Some weeks later the official report of autopsy showed both old and acute confluent subcutaneous hemorrhages on the surface of both arms, trunk, and limbs (estimated in between 1.5 and $17.4 \mathrm{~cm}$ in diameter at the autopsy). No signs of trauma could be established. A left sided large confluent hemorrhage with cerebral herniation was also detected. Furthermore intraperitoneal hemorrhage was also observed, it was estimated $900 \mathrm{ml}$ but no clear loci for bleeding were identified (i.e., malignancy, vascular involvement).

\section{Discussion}

Cerebral hemorrhage is a rare event in AHA but described in literature [2]. In our opinion the characteristics and the severity of the non-traumatic cutaneous hematoma (Fig. 1) could not be related exclusively to anti-platelet therapy, especially low-dose acetylsalicylic acid [3], in the present case. Given the absence of previous bleeding events, both traumatic or spontaneous, and the initial clinical picture during such an antithrombotic therapy a platelet count and basic coagulation tests (PT, aPTT and fibrinogen) should be investigated to detect a putative acquired coagulopathy or hemostasis defect.

Furthermore, in order to the low incidence of cerebral hemorrhage in AHA, we cannot rule out, in the present case, that the concomitant use of aspirin may have increased the hemorrhage risk, derived mostly from the acquired coagulopathy, resulting in a more severe and then fatal case.

In conclusion, we believe that this case renews the opportunity to consider already in the emergency-urgency setting the possibility of acquired coagulative disorders, other than pharmacological induced, in patients with spontaneous bleeding in absence of a previous bleeding history and not immediately attributable to a certain cause. In this setting at least basic coagulative tests such as platelet count, PT, aPTT and fibrinogen should be always warranted.
This case could be of particular interest for daily medical practice because this presentation is less rare than is often considered [4] and the diagnosis can be suspected with simple basic coagulation tests, then directing the patient to specialized examinations for the definition and relative management that it can guarantee, fortunately in the majority of cases, a good outcome [4].

Author contributions LP: conception and design of the manuscript, drafting of the manuscript and reference collection. FB: contributed to the draft of the manuscript, PC contributed to laboratory diagnostic tests, AS contributed to data and reference collection, MB supervised the manuscript and revised it critically.

Funding Each author approved the final version of the manuscript.

\section{Declarations}

Conflict of interest The authors declare that they have no conflict of interest.

Human and animal rights This article does not contain any study with human and animals performed by any of the authors.

Informed consent Not applicable.

\section{Reference}

1. Knoebl P, Marco P, Baudo F (2012) European acquired haemophilia registry (EACH2). Demographic and clinical data in acquired hemophilia a: results from the European Acquired Haemophilia Registry (EACH2). J Thromb Haemost 10:622-631

2. Franchini M, Vaglio S, Marano G (2017) Acquired hemophilia a: a review of recent data and new therapeutic options. Hematology 22:514-520

3. Zhu H, Xu X, Fang X, Ying F, Song L, Gao B, Tong G, Zhou L, Chen T (2021) Efficacy and safety of long-term antithrombotic strategies in patients with chronic coronary syndrome: a network meta-analysis of randomized controlled trials. J Am Heart Assoc. https://doi.org/10.1161/JAHA.120.019184

4. Zanon E, Pasca S, Borchiellini A, Lodigiani C, Molinari AC, Ambaglio C, Valeri F, Preti PS, Moscatelli P, Simioni P (2020) Susoctocog-alfa $\left(\right.$ Obizur $\left.^{\circledR}\right)$ in the treatment of nine elderly patients with acquired haemophilia A: an Italian multicentre real world experience. Blood Transfus. 18:312-321. https://doi.org/10.2450/ 2020.00006-20

Publisher's Note Springer Nature remains neutral with regard to jurisdictional claims in published maps and institutional affiliations. 